\title{
Photoexcitation of bulk polarons in rutile $\mathrm{TiO}_{2}$
}

\author{
Alex J. Tanner $\odot,{ }^{1,2}$ Bo Wen $\odot,{ }^{3}$ Yu Zhang $\odot,{ }^{1,2, *}$ Li-Min Liu, ${ }^{4}$ Helen H. Fielding $\odot,{ }^{1}$ \\ Annabella Selloni $\odot,^{3}$ and Geoff Thornton $\oplus^{1,2, \dagger}$ \\ ${ }^{1}$ Department of Chemistry, University College London, 20 Gordon Street, London WC1H OAJ, United Kingdom \\ ${ }^{2}$ London Centre for Nanotechnology, University College London, 17-19 Gordon Street, London WC1H 0AH, United Kingdom \\ ${ }^{3}$ Department of Chemistry, Princeton University, Princeton, New Jersey 08540, USA \\ ${ }^{4}$ School of Physics, Beihang University, Beijing 100083, China
}

(Received 27 July 2020; revised 9 February 2021; accepted 15 February 2021; published 24 March 2021)

\begin{abstract}
The excitation of surface-localized polaronic states has recently been discussed as an additional photocatalytic channel to band gap excitation for rutile $\mathrm{TiO}_{2}$. A contribution from photoexcitation of bulk polarons could, in principle, provide a greater contribution because of their higher number and their protection from oxidation. However, determining such a contribution to the photoyield is challenging and has not been achieved thus far. Here we use two photon photoemission spectroscopy measurements to separate bulk and surface polaron photoexcitation. We find that bulk polarons are less bound by $0.2 \mathrm{eV}$ compared with polarons at the surface, consistent with our results of hybrid density functional theory calculations. Because the excited state is also shifted to higher energy, bulk polarons have the same photoexcitation resonance energy as at the surface $(3.6 \mathrm{eV})$ with a threshold at $3.1 \mathrm{eV}$. This is degenerate with the band gap, suggesting that bulk polarons could also provide an additional contribution to the photoyield.
\end{abstract}

DOI: 10.1103/PhysRevB.103.L121402

There is considerable interest in $\mathrm{TiO}_{2}$ as a material for photocatalysis due to its stability, natural abundance, and low cost. In the long-accepted picture of $\mathrm{TiO}_{2}$ photocatalysis, electrons and holes created via band gap photoexcitation in the depletion or accumulation layer are swept to surfaces where they enable redox processes [1-3]. Recently, state selective studies using two photon photoemission (2PPE) have identified an additional photoexcitation mechanism at the surface of rutile $\mathrm{TiO}_{2}(110)$ [4-11]. This process involves the excitation of polaronic excess electrons in surface-localized band-gap states (BGS) to conduction band states. These surface BGS arise largely from bridging oxygen vacancies $\left(\mathrm{O}_{\mathrm{vac}}\right)$ [12], which remain at the same energy after the vacancies react with water to form bridging hydroxyls $\left(\mathrm{OH}_{\mathrm{b}}\right)$ (see Fig. 1) [13]. In 2PPE, the onset for photoexcitation from the BGS to the conduction band states is $3.1 \mathrm{eV}[4,6]$, which coincides with the optical band gap. How much of a role this excitation channel plays in photocatalysis is not known; however, it is known that the active photoexcitation depth for rutile photocatalysis extends to $2.5 \mathrm{~nm}$ [14], suggesting that the surface-localized component is likely to be small.

Polarons in bulk rutile $\mathrm{TiO}_{2}$ have a different origin from those at the surface, arising mostly from impurities and $\mathrm{Ti}$ interstitials $\left(\mathrm{Ti}_{\text {int }}\right)$ resulting from reduction (see Fig. 1). This gives rise to $n$-type semiconductor behavior. Vacuum annealing creates interstitials in $\mathrm{TiO}_{2}$, where excess electrons

\footnotetext{
${ }^{*}$ Current address: Central Laser Facility, STFC Rutherford Appleton Laboratory, Harwell Campus, Didcot OX11 0QX, United Kingdom.

${ }^{\dagger}$ Corresponding author: g.thornton@ucl.ac.uk
}

localize as polarons. It has been established that this leads to an increase in the photocatalytic activity, an important motivation to further investigate the role of polarons [14]. In light of the results from studies of surface-localized BGS, it is interesting to explore whether polarons in the bulk may also offer an alternative photoexcitation channel to band gap excitation. However, bulk polarons are difficult to study experimentally, in part due to the surface sensitivity of state-resolved techniques. In this Letter we describe a study that addresses this challenge.

Electron polarons in $\mathrm{TiO}_{2}$ lie in a pseudo-octahedral crystal field. In $2 \mathrm{PPE}$ of the $\mathrm{TiO}_{2}(110)$ surface, polarons are excited to an intermediate state $\sim 2.6 \mathrm{eV}$ above the Fermi energy, $E_{F}[4,6,7,10,15]$. It is known from density functional theory (DFT) calculations that the intermediate state is $t_{2 g}$ in character, with photoexcitation representing a $t_{2 g} \rightarrow t_{2 g}$ transition within the distorted octahedral crystal field of the BGS electrons [7]. The 2PPE intensity is dependent upon the polarization of the incident light and its maximum occurs when the electric field vector of the light is perpendicular to the [001] azimuth. The 2PPE intensity also increases with $\mathrm{OH}_{\mathrm{b}}$ concentration. UV photoemission spectroscopy (UPS) and DFT show that dissociated water both draws polarons to the surface and changes the $d$ electron character to $d_{x y}$. This couples more effectively with the $\mathrm{Ti}^{3+}$ excited state in the conduction band $[4,6,10,13,15]$.

$\mathrm{TiO}_{2}(110)$ is usually hydroxylated in UHV, protecting the surface BGS [16]. However, exposure to $\mathrm{O}_{2}$ removes these electrons, with BGS polarons only remaining in the selvedge and bulk [17-20]. These can be accessed by 2PPE since the probing depth is about $5 \mathrm{~nm}$ based on measurements of one-photon photoemission [21]. This is much larger than the 


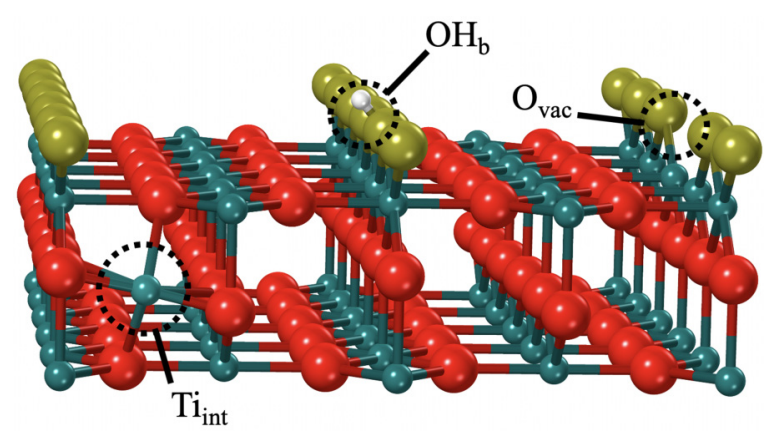

FIG. 1. Ball and stick model of rutile $\mathrm{TiO}_{2}(110)$. Green spheres denote $\mathrm{Ti}$ ions. Red spheres represent $\mathrm{O}$ ions, with twofold coordinated bridging $\mathrm{O}$ shaded yellow. White sphere signifies hydrogen. Common defects of interstitial titanium atoms $\left(\mathrm{Ti}_{\text {int }}\right)$, bridging hydroxyls $\left(\mathrm{OH}_{\mathrm{b}}\right)$ and oxygen vacancy $\left(\mathrm{O}_{\mathrm{vac}}\right)$ are labeled, which represent locations of polaron localization.

probing depths common in UPS ( $1 \mathrm{~nm})[12,17]$. Figure 2(a) show the 2PPE signal from rutile $\mathrm{TiO}_{2}(110)$ BGS electrons for three different terminations. Reduced, partially hydroxylated, and oxidized $\mathrm{TiO}_{2}(110)$ are denoted as R-R110, $\mathrm{H}_{\mathrm{p}}-$ R110, and O-R110, respectively. All spectra were recorded with a pump and probe photon wavelength of $350 \mathrm{~nm}$ $(3.54 \mathrm{eV})$, with the sample orientation such that the [001] azimuth is perpendicular to the plane of incidence (All experimental details can be found in the Supplemental Material (SM) [22]).

The three terminations (R-R110, $\mathrm{H}_{\mathrm{p}}-\mathrm{R} 110$, and O-R110) were produced consecutively in situ to reduce uncertainties in measurement variables such as changes in the laser power. An example of spectral progression throughout an entire experiment is shown in the SM [22], Fig. S1. The R-R110 2PPE spectrum was recorded after the sample cooled to $300 \mathrm{~K}$ following sample preparation (detailed in the SM [22]). Accurate monitoring of the sample temperature is important as temperature is known to affect 2PPE spectra of $\mathrm{TiO}_{2}$ [23]. Surface $\mathrm{O}_{\text {vac }}$ will readily react with residual UHV water; however, the chamber base pressure of $1 \times 10^{-10}$ mbar ensures the slow rate of hydroxylation within the time required to record the R-R110 spectrum in Fig. 2(a). He-II (40.8 eV) UPS showed no observable peak corresponding to the $3 \sigma \mathrm{OH}$ orbital at $11.0 \mathrm{eV}$ binding energy [4,17] (see the SM [22] Fig. S2).

At longer times, the rutile $\mathrm{TiO}_{2}(110)$ sample gradually hydroxylates from residual water in the UHV chamber. Hydroxylation is monitored by the decrease in work function $(\phi)$ with $2 \mathrm{PPE}$ and from the increase in the $3 \sigma$ peak in the UPS He-II spectrum. The $\mathrm{H}_{\mathrm{p}}-\mathrm{R} 110$ spectrum in Fig. 2(a) was recorded after the sample had been exposed to the residual UHV vacuum for approximately $90 \mathrm{~min}$. The increased intensity of the resonance upon hydroxylation is understood within the framework of BGS electron redistribution towards the surface $[4,13,15]$. At this stage the sample is partially hydroxylated at $\sim 0.05 \mathrm{ML}$ ( 1 monolayer corresponds to the number of surface unit cells). It had been assumed that $\mathrm{OH}_{\mathrm{b}}$ causes an increase in 2PPE intensity due to the stabilization of photoexcited electrons from $\mathrm{H}$ atom related $s$ orbitals [11]. As noted above, it is now understood that electrons redistribute to the surface and undergo an orbital character change to $d_{x y}$, which enhances the $t_{2 g} \rightarrow t_{2 g}$ oscillator strength.

The sample was then exposed to $\mathrm{O}_{2}$ at a pressure of $2 \times$ $10^{-8} \mathrm{mbar}$. This resulted in an immediate increase in $\phi$ and an immediate decrease in the 2PPE resonance. $\mathrm{As}_{2}$ is dosed, it primarily adsorbs at remaining $\mathrm{O}_{\mathrm{vac}}$ and five-coordinate $\mathrm{Ti}$ rows, while also reacting with $\mathrm{OH}_{\mathrm{b}}[17,24]$. This oxidation process decreases the 2PPE resonance by removing surface and subsurface BGS [17]. The O-R110 spectrum in Fig. 2(a) was recorded following exposure to $54 \mathrm{~L}$ (1 Langmuir = $1.32 \mathrm{mbar} s$ ) of $\mathrm{O}_{2}$. Corresponding UPS and 1PPE spectra also show the removal of BGS (see the SM [22] Fig. S3). The 2PPE spectra evidence a blue shift in the resonance energy by $\sim 0.15 \mathrm{eV}$ following removal of the surface BGS electrons.

2PPE can arise from an incoherent process (two sequential one-photon excitations via an intermediate state) or a coherent process (simultaneous two-photon excitation of an occupied state) [25]. A plot of photon energy (eV) vs final state electron energy $E-E_{F}(\mathrm{eV})$ results in a gradient of 1 or 2 for incoherent and coherent processes, respectively, with a $y$ intercept that is equal to the intermediate or initial state energy, respectively. In the 2PPE spectra of $\mathrm{TiO}_{2}(110)$, it has been shown that both processes contribute to the $2 \mathrm{PPE}$ yield. At the $2 \mathrm{PPE}$ resonant photon energy $\left[3.54 \mathrm{eV}, 350 \mathrm{~nm}\right.$ for $\left.\mathrm{TiO}_{2}(110)\right]$ there is increased intensity in the 2PPE signal as the peak is a sum of the incoherent and coherent features [4,25]. 2PPE with higher photon energies separates the two features, allowing for peak fitting analysis and the isolation of individual contributions. To achieve this, the 2PPE background is modeled and the peaks fit with Gaussians (see the SM [22] Fig. S4, including Refs. $[4,6,7,10,11])$. Figure 2(b) shows a plot of $h v$ (eV) vs $E-E_{F}(\mathrm{eV})$ for the incoherent 2PPE from O-R110 and H-R110, with H-R110 being a surface hydroxylated in residual UHV for $\sim 48 \mathrm{~h}$ to ensure full occupation of $\mathrm{O}_{\text {vac }}$ $(\sim 0.1 \mathrm{ML})$ with $\mathrm{OH}$. This will remain stable throughout the 2PPE measurements $[19,26]$. O-R110 spectra were recorded following a subsequent saturation exposure to $\mathrm{O}_{2}$, with $\mathrm{P}_{\mathrm{O}_{2}}$ being maintained at $2 \times 10^{-9}$ mbar to prevent step-edge hydroxylation $[27,28]$. Figure 2 (b) shows that the intermediate state in O-R110 lies $0.14 \mathrm{eV}$ higher in energy than that for H-R110.

To assist our interpretation of the experimental observations, we employ DFT calculations using the HSE06 hybrid functional [29], which is known to describe polaron states in $\mathrm{TiO}_{2}$ with good accuracy [7]. The partial density of states (PDOS) and oscillator strengths were calculated for polarons arising from $\mathrm{OH}_{\mathrm{b}}, \mathrm{O}_{\mathrm{vac}}$, and $\mathrm{Ti}_{\text {int }}$ at the rutile $\mathrm{TiO}_{2}(110)$ surface (modeled using a 6 trilayer slab). The method details for the calculations are shown in the SM [22] (see also Refs. [29-32] therein). For the case of $\mathrm{Ti}_{\text {int }}$, the location was varied from the immediate subsurface (L1) to two (L2) and three (L3) layers below the surface (see the SM [22] Fig. S5). These different locations represent the behavior of polarons in surface and bulk-like environments. Figures 2(c), 2(d), and 2(e) show the PDOS of rutile $\mathrm{TiO}_{2}(110)$ surfaces with $\mathrm{O}_{\text {vac }}$, $\mathrm{OH}_{\mathrm{b}}$, and (L3) $\mathrm{Ti}_{\text {int }}$, respectively. The polarons towards the surface $\left(\mathrm{O}_{\mathrm{vac}}\right.$ and $\left.\mathrm{OH}_{\mathrm{b}}\right)$ lie at slightly lower energies (i.e., deeper in the band gap) than those in the bulk [(L3) $\left.\mathrm{Ti}_{\text {int }}\right]$, as generally expected [33]. This is mirrored in the trend of the BGS PDOS of $\mathrm{Ti}_{\text {int }}$ located at L1, L2, and L3 in the 
(a)

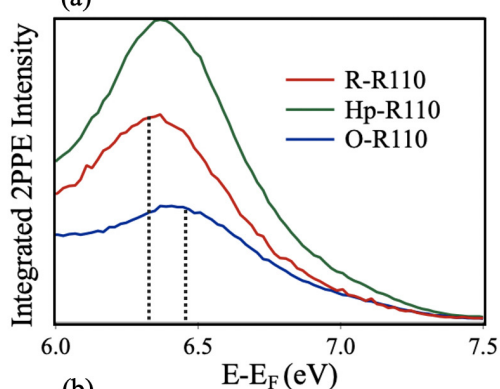

(b)

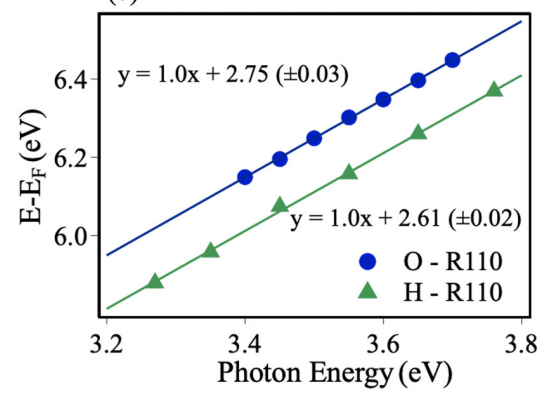

(c) (Surface) $\mathrm{O}_{\mathrm{vac}}$

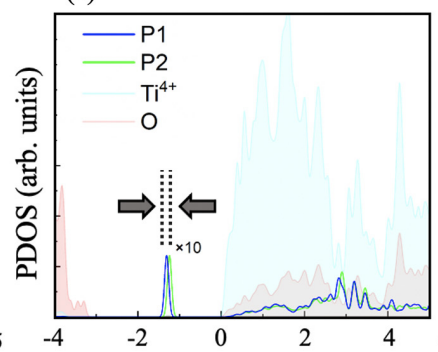

(f)

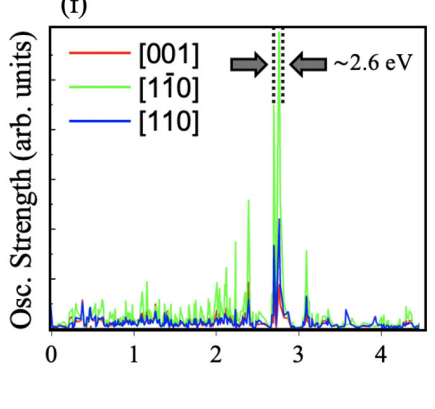

(d) (Surface) $\mathrm{OH}_{\mathrm{b}}$

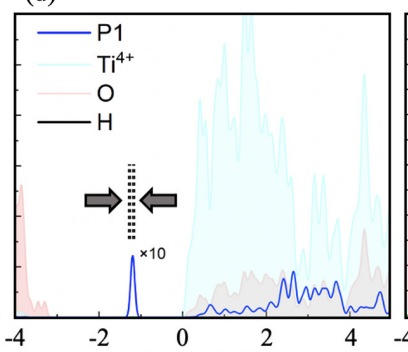

(g)

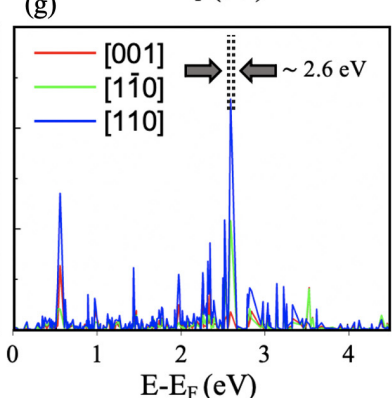

(e)

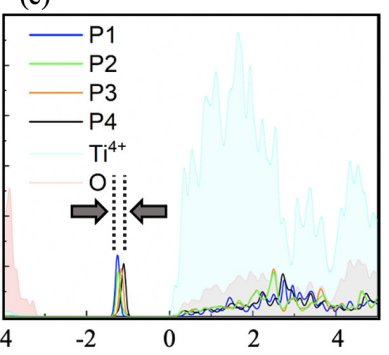

(h)

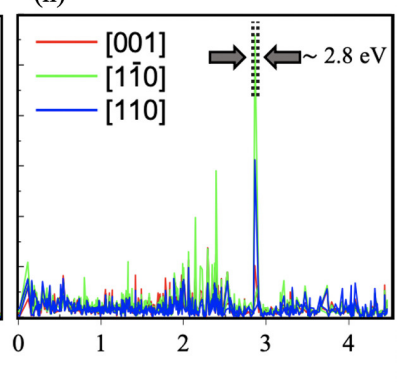

FIG. 2. (a) 2PPE spectra from R-R110, $\mathrm{H}_{\mathrm{p}}-\mathrm{R} 110$, and O-R110, given by red, green, and blue lines respectively, recorded with $p$-polarized $3.54 \mathrm{eV}(350 \mathrm{~nm})$ light, with the [001] azimuth perpendicular to the plane of incidence. The prominent feature in the spectra arises from an initial $d \rightarrow d$ transition within orbitals of $t_{2 g}$ symmetry [7]. Dashed lines indicate the peak maxima. The spectra are normalized to the peak at the work-function cutoff. (b) Comparison between the H-R110 and O-R110 photon energy dependence of the incoherent 2PPE feature. The H-R110 plot contains photon energies 3.76-3.26 eV (330-380 nm) and the O-R110 plot 3.70 - 3.40 eV(335-365 nm) according to the equation: $E-E_{F}=h v_{\text {probe }}+E_{\text {intermediate }}$. (c), (d), and (e) show the calculated PDOS for Ti ${ }^{3+}$ ions induced by $\mathrm{O}_{\text {vac }}, \mathrm{OH}_{\mathrm{b}}$ and $\mathrm{Ti}_{\text {int }}(\mathrm{L} 3)$, respectively, in $\mathrm{R} 110$. The polaron traces are magnified by a factor of 10 relative to the PDOS for $\mathrm{Ti}^{4+}$ and $\mathrm{O}$ (pale blue and pink shadings). Dashed lines and arrows represent the width of the occupied BGS. PDOS are decomposed into individual polarons, denoted by "P" label (see

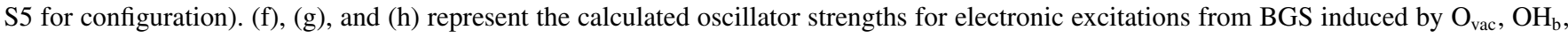
and $\mathrm{Ti}_{\text {int }}(\mathrm{L} 3)$ to the conduction band. Dashed lines and arrows represent the width and location of maximum transitions. Transitions with the

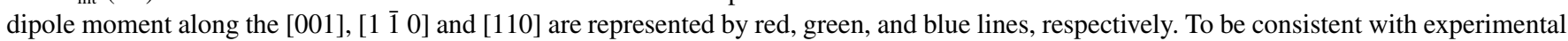
observation, the Fermi energies in (c)-(h) are rescaled to the conduction band minima.

pristine surface (see the SM [22] Fig. S6). At L3, polarons are degenerate around a single peak $\sim 1.2 \mathrm{eV}$ below $E_{F}$, however at $\mathrm{L} 2$ the distribution splits into two peaks at $\sim 1.1$ and $1.4 \mathrm{eV}$ below $E_{F}$. At L1, the peaks are separated further with one at $\sim 1.5 \mathrm{eV}$, and the other $\sim 0.9 \mathrm{eV}$ below $E_{F}$, reflecting the more diverse coordination environments of polarons in proximity to the surface. To support these findings, we further varied the location of the polarons around $\mathrm{Ti}_{\text {int }}$ and $\mathrm{O}_{\mathrm{vac}}$ (see the SM [22] Fig. S7, including Refs. [18,34]). While the number of possible polaron configurations is very large $[18,34]$ and investigating all of them is beyond the scope of this work, all the results of our calculations confirm that the energy levels of polarons localized at bulk sites are closer to $E_{F}$ than those of surface polarons (see the SM [22] Fig. S7, including Refs. [18,34]).

The oscillator strengths of electron transitions from BGS associated with $\mathrm{O}_{\text {vac }}, \mathrm{OH}_{\mathrm{b}}$, and $\mathrm{Ti}_{\text {int }}$ (L3) are shown in Figs. 2(f), 2(g), and 2(h), respectively. The contributions are separated into the directions of the transition dipole moments according to the sample azimuths. The stabilization of the occupied states in Figs. 2(c) and 2(d) is largely reflected by a shift in peak oscillator strengths from $2.8 \mathrm{eV}$ for (L3) $\mathrm{Ti}_{\text {int }}$ to $2.6 \mathrm{eV}$ for $\mathrm{O}_{\mathrm{vac}}$ and $\mathrm{OH}_{\mathrm{b}}$. The oscillator strengths were also calculated for $\mathrm{Ti}_{\text {int }}$ at $\mathrm{L} 1$ and $\mathrm{L} 2$ and evidence the same surface to bulk trend (see the SM [22] Figs. S6, S8). Prior to dosing
$\mathrm{O}_{2}$, the 2PPE signal is a sum of contributions from polarons in the surface, subsurface and bulk. However, after exposure to $54 \mathrm{~L}$ of $\mathrm{O}_{2}$ only bulk polarons contribute to the spectra. Both the experimental data and the calculations show the associated intermediate states lie at $0.14-0.20 \mathrm{eV}$ to higher energy for the bulk polarons.

From the discussion above, the 2PPE resonance in R- and H-R110 will have four features, with incoherent and coherent components from the surface and bulk. This is demonstrated with peak fitting in Fig. 3(a). We determine the positions and heights of the incoherent $(i)$ peaks from a comparison of H-R110 and O-R110 spectra (see the SM [22] Fig. S9), with those for the coherent peaks $(c)$ determined from 2PPE spectra taken with higher photon energy (see the SM [22] Fig. S10). The variation of the integrated intensity of the 2PPE resonance recorded from $\mathrm{H}$ - and $\mathrm{O}-\mathrm{R} 110$ show that both have a maximum at $3.54 \mathrm{eV}$, indicating that the initial excitation state is also $0.2 \mathrm{eV}$ lower in energy at the surface and the splitting of the $t_{2 g}$-like states remains constant [see Fig. 3(b)]. The calculated excitation energy $(\sim 3.8 \mathrm{eV})$ is larger than that observed experimentally. This discrepancy can be attributed to the approximate models employed and the inherent limitations of DFT in the description of excitations. This is illustrated by the fact that our HSE06 calculations give a 3.4-eV band gap for the $\mathrm{TiO}_{2}(110)$ surface, rather than $3.1 \mathrm{eV}$. The excitation 

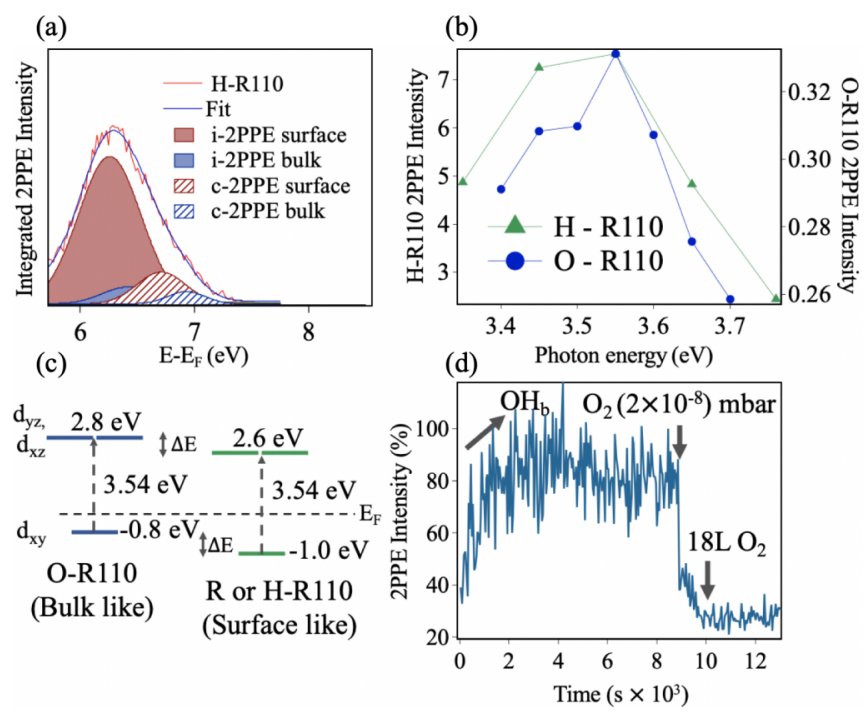

FIG. 3. (a) $2 \mathrm{PPE}(3.65 \mathrm{eV}, 340 \mathrm{~nm})$ spectrum from $\mathrm{H}-\mathrm{R} 110$ fitted with Gaussian distributions showing the incoherent (i) and coherent (c) contributions of the bulk and surface features at this photon energy. (b) Plot of peak intensity as a function of photon energy for H-R110 and O-R110. The peak intensity represents a convolution of both coherent and incoherent $2 \mathrm{PPE}$ processes. The spectra are normalized at the work function cut off. Corresponding data for R$\mathrm{R} 110$ are not shown since this surface reacts with residual $\mathrm{H}_{2} \mathrm{O}$ over the period of the $2 \mathrm{PPE}$ measurements with varying photon energy. (c) Schematic of the excitations in bulk and surface environments on the basis of calculations and 2PPE spectra as well as previously published data [7]. (d) Time evolution of the rutile (110) 2PPE peak monitored over the course of $200 \mathrm{~min}$. There is an immediate decrease in intensity when the surface is exposed to $\mathrm{O}_{2}$.

scheme of polarons at the $\mathrm{TiO}_{2}(110)$ surface and bulk is shown in Fig. 3(c).

Figure 3(d) shows the time variation of the raw 2PPE resonance intensity as the surface is first exposed to the residual vacuum and then to $\mathrm{O}_{2}$. The maximum intensity observed (100\%) corresponds to $\mathrm{H}_{\mathrm{p}}-\mathrm{R} 110$, as also seen in Fig. 2(a). The intensity is seen to increase with $\mathrm{OH}_{\mathrm{b}}$ coverage and decrease rapidly after exposure to $\mathrm{O}_{2}$, stabilizing at $25 \%$ of the $\mathrm{H}_{\mathrm{p}}-\mathrm{R} 110$ intensity. This clearly evidences the surface dominance of the resonance. This $18 \mathrm{~L}$ exposure to $\mathrm{O}_{2}$ is significantly higher than that used in previous studies, which likely explains the conclusion in earlier work that the intense resonance arises from up to $4 \mathrm{~nm}$ into the bulk [11]. We note that UPS and 1PPE spectra recorded after a lower $\mathrm{O}_{2}$ exposure (7 L) (see the SM [22] Fig. S3) still display a significant surface BGS intensity.

In summary, we have identified the depth-dependent photoexcitation channels of polarons in rutile $\mathrm{TiO}_{2}(110)$ through the use of 2PPE and DFT. 2PPE spectra of reduced and hydroxylated $\mathrm{TiO}_{2}(110)$ are dominated by photoexcitation of surface polarons. Nevertheless, photoexcitation of bulk polarons can be distinguished after oxidizing the surface to remove the surface component. This occurs at the same photon energy as at the surface, where both the initial and excited states lie at about $0.2 \mathrm{eV}$ higher energy than at the surface. These photoexcitation events in the bulk are more likely to contribute to photocatalytic processes simply because of the greater number of polarons arising from Ti interstitials. Control over the local environment of polarons may be key for future catalyst design as we seek to exploit their role in photocatalytic applications.

We thank R. Riley, O. Tau, and R. Kerr for their assistance in maintaining stable laser and UHV conditions. This work was supported by the European Research Council Advanced Grant ENERGYSURF (G.T.), EPSRC (U.K.) (EP/N509577/1), and the Royal Society (U.K.) through a Wolfson Research Merit Award to G.T. The research is supported by the TIGRESS high performance computer center at Princeton University. B.W. and A.S. acknowledge the support of DoE-BES, Division of Chemical Sciences, Geosciences and Biosciences under Award No. DESC0007347. L.M.L. was supported by the National Natural Science Foundation of China (No. 51861130360 and No. 11974037) and a Royal Society Newton Advanced Fellowships (No. NAF/R1/180242).

The authors declare no competing financial interest.
[1] A. Fujishima and K. Honda, Nature (London) 238, 37 (1972).

[2] A.L. Linsebigler, G. Lu, and J. T. Yates, Chem. Rev. 95, 735 (1995).

[3] J. Schneider, M. Matsuoka, M. Takeuchi, J. Zhang, Y. Horiuchi, M. Anpo, and D. W. Bahnemann, Chem. Rev. 114, 9919 (2014).

[4] Y. Zhang, D. T. Payne, C. L. Pang, H. H. Fielding, and G. Thornton, J. Phys. Chem. Lett. 6, 3391 (2015).

[5] Y. Zhang, D. T. Payne, C. Pang, C. Cacho, R. T. Chapman, E. Springate, H. H. Fielding, and G. Thornton, J. Phys. Chem. Lett. 10, 5265 (2019).

[6] Z. Wang, B. Wen, Q. Hao, L.-M. Liu, C. Zhou, X. Mao, X. Lang, W.-J. Yin, D. Dai, A. Selloni, and X. Yang, J. Am. Chem. Soc. 137, 9146 (2015).

[7] B. Wen, Q. Hao, W.-J. Yin, Le Zhang, Z. Wang, T. Wang, C. Zhou, A. Selloni, X. Yang, and L.-M. Liu, Phys. Chem. Chem. Phys. 20, 17658 (2018).
[8] D. T. Payne, Y. Zhang, C. L. Pang, H. H. Fielding, and G. Thornton, Surf. Sci. 652, 189 (2016).

[9] D. T. Payne, Y. Zhang, C. L. Pang, H. H. Fielding, and G. Thornton, Top. Catal. 60, 392 (2016).

[10] A. Argondizzo, X. Cui, C. Wang, H. Sun, H. Shang, J. Zhao, and H. Petek, Phys. Rev. B 91, 155429 (2015).

[11] A. Argondizzo, S. Tan, and H. Petek, J. Phys. Chem. C 120, 12959 (2016).

[12] C. M. Yim, C. L. Pang, and G. Thornton, Phys. Rev. Lett. 104, 036806 (2010).

[13] C. M. Yim, J. Chen, Y. Zhang, B.-J. Shaw, C. L. Pang, D. C. Grinter, H. Bluhm, M. Salmeron, C. A. Muryn, A. Michaelides, and G. Thornton, J. Phys. Chem. Lett. 9, 4865 (2018).

[14] T. Luttrell, S. Halpegamage, J. Tao, A. Kramer, E. Sutter, and M. Batzill, Sci. Rep. 4, 4043 (2014). 
[15] B. Wen, W.-J. Yin, A. Selloni, and L.-M. Liu, J. Phys. Chem. Lett. 9, 5281 (2018).

[16] K. I. Hadjiivanov and D. G. Klissurski, Chem. Soc. Rev. 25, 61 (1996).

[17] S. Wendt, P. T. Sprunger, E. Lira, G. K. H. Madsen, Z. Li, J. Ø. Hansen, J. Matthiesen, A. Blekinge-Rasmussen, E. Lægsgaard, B. Hammer, and F. Besenbacher, Science 320, 1755 (2008).

[18] K. Morita, T. Shibuya, and K. Yasuoka, J. Phys. Chem. C 121, 1602 (2017).

[19] C. L. Pang, R. Lindsay, and G. Thornton, Chem. Soc. Rev. 37, 2328 (2008).

[20] U. Diebold, Surf. Sci. Rep. 48, 53 (2003).

[21] D. P. Woodruff, Modern Techniques of Surface Science (Cambridge University Press, Cambridge, 2016).

[22] See Supplemental Material at http://link.aps.org/supplemental/ 10.1103/PhysRevB.103.L121402 for experimental details, computational details, supporting DFT calculations and further photoemission spectra.

[23] H. Shang, A. Argondizzo, S. Tan, J. Zhao, P. Rinke, C. Carbogno, M. Scheffler, and H. Petek, Phys. Rev. Res. 1, 033153 (2019).

[24] O. Bikondoa, C. L. Pang, R. Ithnin, C. A. Muryn, H. Onishi, and G. Thornton, Nat. Mater. 5, 189 (2006).
[25] H. Ueba and B. Gumhalter, Prog. Surf. Sci. 82, 193 (2007).

[26] Michael A. Henderson, William S. Epling, A. Charles H. F. Peden, and C. L. Perkins, J. Phys. Chem. B 107, 534 (2002).

[27] H. H. Kristoffersen, J. O. Hansen, U. Martinez, Y. Y. Wei, J. Matthiesen, R. Streber, R. Bechstein, E. Lægsgaard, F. Besenbacher, B. Hammer, and S. Wendt, Phys. Rev. Lett. 110, 146101 (2013).

[28] U. Martinez, L. B. Vilhelmsen, H. H. Kristoffersen, J. Stausholm-Møller, and B. Hammer, Phys. Rev. B 84, 205434 (2011).

[29] J. Heyd, G. E. Scuseria, and M. Ernzerhof, J. Chem. Phys. 118, 8207 (2003).

[30] J. VandeVondele, M. Krack, F. Mohamed, M. Parrinello, T. Chassaing, and J. Hutter, Comput. Phys. Commun. 167, 103 (2005).

[31] S. Goedecker, M. Teter, and J. Hutter, Phys. Rev. B 54, 1703 (1996).

[32] D. Pan, Q. Wan, and G. Galli, Nat. Commun. 5, 3919 (2014).

[33] C. Di Valentin and A. Selloni, J. Phys. Chem. Lett. 2, 2223 (2011).

[34] N. A. Deskins, R. Rousseau, and M. Dupuis, J. Phys. Chem. C 115, 7562 (2011). 